\title{
Nutrition, alcohol and drug abuse
}

By R. C. Denney, School of Chemistry, Thames Polytechnic, London SE $186 P F$ and R. Johnson, The Medical Council on Alcoholism, 3 Grosvenor Crescent, London $S W_{\mathrm{I}} X_{7} E E$

Alcohol (ethanol) is the commonest addictive substance known to man and is accepted as a legal psychotropic drug by most customs and religions. It is, however, well known that heavy consumption of alcohol will lead to physical, mental, social and economic damage, just as it will with the chronic consumption of any other pharmacologically active substance. It is because of this and the increased use of all forms of drugs that the whole matter of drug use, abuse and addiction has become one of the major preoccupations of the World Health Organization. Its Expert Committee on Drugs Liable to Produce Addiction has now been working for 25 years to co-ordinate international action to control the spread of drug abuse.

It is, however, essential to start with a clear definition of addiction, and that of the World Health Organization (1957) is more than adequate for this purpose: 'Drug addiction is a state of periodic or chronic intoxication detrimental to the individual and society, produced by the repeated consumption of a drug (natural or synthetic). Its characteristics include: (I) an overpowering desire or need (compulsion) to continue taking the drug and to obtain it by any means; (2) a tendency to increase the dose; (3) a psychological and sometimes a physical dependence on the effects of the drug'.

This definition does not seek to differentiate between the various types of drugs, but it should be clearly understood that alcohol differs considerably from virtually every other drug, although its withdrawal symptoms are said to be similar to those for barbiturates. The very fact that it is socially acceptable makes alcohol a very insidious drug and it frequently becomes the starting point for other forms of drug dependency. Alcohol is the most widely misused and abused drug of all, but three-quarters of all alcoholics are able to hold down jobs and there is a strong tendency for alcohol-dependent people to be protected and tolerated by society. As a result, the full magnitude of the alcohol problem is usually obscured while problems of other forms of drug abuse are frequently more obvious.

Contrary to popular belief, alcohol is a depressant, not a stimulant. After being absorbed by the walls of the duodenum and upper intestine it rapidly affects the central nervous system, numbing the high reasoning centres of the brain. Whilst this creates a sense of relaxation which may show itself in a display of conviviality and a lessening of inhibitions, the progressive increase of the blood alcohol level results in a disruption of the motor skills such that reaction times and co-ordination deteriorate. 
From a nutritional point of view, alcohol has one major difference from other drugs in that it is a food. Weight-for-weight it provides almost as much energy as does fat, and produces the familiar 'drinker's gut' if there is no corresponding reduction in normal food intake. Just 2 pints of beer can provide over $1464 \mathrm{~kJ}$ ( $35^{\circ}$ $\mathrm{kcal}$ ) and, as a result, a person drinking 6 pints of beer each day is obtaining nearly half his daily requirement of energy from alcohol. No other drug has this effect. The consequence of this for the heavy drinker is that he (or she) eats less and less frequently until eventually for the alcoholic the craving for alcohol will almost totally replace the desire to eat.

Lieber ( 1980 ) has referred to alcoholic drinks as providing 'empty calories' because, although they provide energy, they lack the nutritional balance of proteins, vitamins and minerals that are essential for the body. This lack contributes eventually, amongst other things, to changes arising in the liver as its main function becomes orientated towards alcohol metabolism. The overall result is cirrhosis of the liver, a weakened heart and a decayed brain. Although deterioration to this extent may take a number of years, it leads to the consequence that the life expectancy of an alcoholic is ro years less than that of the average person.

The malnutrition in the body is further aggravated by the alcohol causing inflammation of the digestive system which in turn impairs the absorption of the nutrients which would normally be available from any food which is eaten. Alcohol, therefore, clearly has a direct influence on the digestive system as well as an indirect influence arising from malnutrition and maldigestion (Denney, 1982). In the case of the heavy drinker, the imbalance is so severe that metabolic pathways are totally disorganized and physical degeneracy sets in, as also occurs with other addictive drugs. The physical effect of this is usually seen in terms of a deterioration of health, a drop in the level of personal care and tidiness and, of course, a general preoccupation with drinking, often carried out in secret.

Many of these more obvious effects, as well as others involving the family and work, are similar to those occurring with other forms of drug addiction. The one important difference is that the alcoholic goes without food partly because he feels he does not need it, whilst other drug addicts go without food mainly because they want to use their money to obtain further supplies of their drugs.

Similarly, all drug addicts unable to obtain a source of supply will, within a short period of time, suffer from withdrawal symptoms. In the case of the alcoholic this will show itself in various forms, such as delirium tremens which is likely to be associated with other disorders arising from vitamin deficiences and poor reflexes. It has, for instance, been suggested that alcoholism is one of the few remaining causes of scurvy and beri-beri in countries with otherwise good nutritional standards, and vitamin deficiency has been implicated as the cause of peripheral neuritis which is common amongst alcoholics. The physical effects of alcohol on the human body may be considered under various headings (Jenkins, 1982):

Liver disease. The extent of liver damage from high alcohol consumption takes several forms, ranging from benign fatty infiltration to alcoholic hepatitis, cirrhosis 
and hepatoma. Cirrhosis is the result of severe alcoholism usually extending over fairly lengthy periods. The theory that cirrhosis arises directly from malnutrition in the heavy drinker rather than from alcohol itself has apparently now been discarded, partly as a result of the work of Lelbach (1972) who demonstrated a correlation between the quantity of alcohol taken daily and the prevalence of cirrhosis.

This does mean that treatment of cirrhosis is more complicated than just restoring a person to a balanced diet. It also requires abstinence from alcohol accompanied by the short-term use of prescribed drugs aided, possibly, by salt restriction and protein restriction.

Heart and circulatory disorders. Alcohol has been shown to be a major cause of damage of heart muscles, but alcoholic cardiomyopathy can be reversed at the preclinical and acute stages by a reduction in alcohol consumption. At the same time it is now generally accepted that moderate drinking can afford some protection against coronary heart disease. Willet et al. (1980) showed a positive relationship between lipoprotein cholesterol levels (which are protective against coronary heart disease) and alcohol consumption. However, a safe level of balance between coronary heart disease and alcoholism does not appear to have been established, but it must be at a fairly low daily intake level, probably of about one or two sherries or I pint of beer.

Disorders of the digestive tract. Excluding liver cancer, it has been found that cancer of the oesophagus is eighteen times more common amongst heavy drinkers than in the population at large. It has also been clearly established that alcohol can damage the linings of the oesophagus, stomach and duodenum causing inflammation and ulceration, as a result of which peptic ulcers have been found to occur in $20 \%$ of people with histories of alcoholism (Royal College of Psychiatrists, 1979).

Brain damage. Over 100 years ago Wernicke ( $188 \mathrm{I}$ ) indicated the importance of alcohol in causing brain damage, and more recent studies have shown an atrophic appearance in the cortex and white matter of young alcoholics, whilst other studies have indicated that there is a reduction in the cerebral blood flow. In some instances nutrition can apparently play an important role in reversing the brain damage, firstly in the provision of a more balanced diet and secondly in treatment with large doses of thiamin. Despite this it is still uncertain whether the deleterious effects of alcohol are due to direct action on the brain or are simply aggravating or reinforcing effects arising due to malnutrition.

Many of the effects of alcohol are paralleled by the action of other drugs which are misused in one way or another. In most instances the overall effect is a general deterioration of the body metabolism due to the action of excessive and continuous use of the drugs, and the malnutrition arising from reduced food intake and/or existence on an inadequately-balanced diet. In many instances the drug addict has already undermined his physical and mental state with his progression from the excessive consumption of alcohol to the abuse of 'harder' drugs. 


\section{Alcohol, drugs and age}

It would be wrong to assume that alcohol abuse and its concomitant nutritional effects only occur with mature adults. The increasing ease of access to alcohol due to home brewing and wider sales in supermarkets and restaurants has led to both a greater consumption of alcohol by the population at large and an earlier experience with alcohol at an increasingly younger age. However, it would be true to say that 'the extent to which conscious intentional alcoholic abuse is prevalent amongst children and adolescents is not known' (National Children's Bureau, I979). What is true is that alcohol is capable of having an effect at the earliest possible age.

Alcohol has been suspected as being responsible for fetal damage for centuries, and in recent years the teratogenic effect of alcohol has become well established (Rosett et al. 1980). Symptoms of the fetal alcohol syndrome observed in the offspring of mothers who drink to excess include: ( 1 ) growth deficiencies in weight, length and brain; (2) facial abnormalities; (3) heart defects; (4) abnormalities in limbs and joints; (5) slowed development and mental deficiency.

The critical time for the fetus appears to be during the third to eighth weeks of the pregnancy, at a time when there is rapid cell division but also when the mother may still not be certain that she is pregnant. As a result there are difficulties in advising women at this stage that they should abstain from alcohol as well as other drugs. Such advice when sought or given is frequently too late to be of maximum value.

Research on other drugs, e.g. thalidomide, indicates that the actual timing of drug intake is crucial with respect to the abnormalities that may occur in the child. In fact, timing may be more crucial than the magnitude of the dose. With thalidomide (Tindle, 1983 ), doses on the thirtieth day of the pregnancy led to abnormalities in both arms and legs, whilst doses on the thirty-fifth day led to only leg deformaties. Critical times such as these have not yet been clearly established for alcohol. However, it is believed that even moderate drinking may lead to spontaneous abortions and physical abnormalities. As a result, the National Council of Women of Great Britain ( 1980 ) has stated that the evidence is now strong enough to justify the statement that 'women who drink during pregnancy may be harming their unborn children'.

It has been reported that alcohol withdrawal symptoms can occur with babies after delivery and that this is characterized by tremors, irritability and overt seizures with mild to moderate mental retardation being quite common. The real causes of these effects are not quite clear, some people attributing them to the ethanol itself, others to acetaldehyde (the first oxidation metabolite of ethanol), whilst some researchers consider that nutritional deficiencies associated with alcoholism of the mother-to-be is a major factor. In any case the view is now held that there is no safe level for alcohol in pregnant women (Smith, $198 \mathrm{r}$; Staisey \& Fried, 1983).

The effects of drugs, particularly alcohol, can be compounded after birth in the case of breast-feeding mothers. Any alcohol passes very rapidly into the breast milk 
and heavy consumption of alcohol by the mother is believed to lead to alcoholic effects in the offspring. Evidence for this is still poorly established, more by lack of data than anything else. Certainly the sleep-inducing effects of alcohol have been well known to nursing mothers of many generations who would have a bottle of stout before feeding their children in order to ensure that both they and the child obtained a good night's sleep.

Young children are coming into contact with alcohol, as well as other drugs and solvents, at an ever younger age with an increasing percentage actually trying alcohol before they even start going to school. Despite this it has been claimed that serious alcohol abuse is uncommon amongst young children (Ritson, 1983) and, whilst they are likely to see the effect of excessive drinking sometimes in their neighbourhood, they are unlikely to do much serious drinking until their teens.

Most teenagers in North America, Europe and Australia are drinking by their mid-teens and, as Hawker (1978) has written in Adolescents and Alcohol, 'With the exception of drunkenness offences and drunken driving, the indices used to estimate the prevalence of problem drinking and alcoholism are difficult to apply to young people, but it is in this area that the figures show a sharp increase over the last five years, particularly in the I 8-2 I age group. Hospital admissions for alcoholism and alcoholic psychosis for young adults have also shown an increase and there have been isolated reports of young people between the ages of 17 and 20 who are receiving treatment for alcoholism'.

Part of this increase may be due to the apparent swing away from other drugs and back to alcohol. However, an added point from Hawker's (1978) survey was that $96 \%$ of $13-16$-year-olds had tried alcohol with almost half of them receiving their first drink at home from their parents, the redeeming aspect of this being that it is probably being consumed under parental guidance. However, this is not necessarily totally good, as Plant et al. (1982) have shown that many $15^{-}$and 16-year-olds in Scotland who were drinking were also suffering from health and social problems. These may, of course, be related to the fact that children of alcoholics are themselves more likely to become alcoholics later in life and the first steps are probably taken whilst they are still in their teens.

Another feature which is becoming apparent from surveys is that the traditional differences between boys and girls are beginning to lessen. Although boys still drink more than girls the gap is narrowing, and girls are using other drugs as much and smoking a little more. As a parallel to this the Parliamentary All-Party Penal Affairs Group ( 1983 ) has reported that the problem of drug misuse for young people, while more limited in scale than alcohol abuse, is rapidly increasing. This is reflected in the fact that the number of addicts of all ages notified to the Home Office as receiving narcotic drugs from doctors in treatment of their addiction on 31 December 1982 was nearly three times the number known at the end of 1972.

Studies on public drunkenness show that the peak incidence rate is in the 1 8-year-old age-group, which coincides with the peak age for alcohol consumption. This is accompanied by all other alcohol-related problems. However, it has been stated that the level of alcohol problems declines with advancing years in line with 
the acceptance of adult responsibilities (Ritson, 1983). Despite this there are factors which serve to indicate if a person is likely to have drinking problems and these are: (1) a family history of alcohol problems; (2) extreme parental views on drinking (either pro or anti); (3) an insecure personality, such as indicated by aggressiveness; (4) a history of childhood or adolescent misbehaviour. Such factors are likely to show themselves in an increased preoccupation with alcohol, minor law offences, periodic accidents, a change in behaviour, apparent regular minor illnesses including complaints of stomach disorders and a dislike of food, and absenteeism.

It would be wrong to minimize either the magnitude of the current problem or of the potential one. Only $6 \%$ of the British adult population are non-drinkers; people drink for experiment, to pretend to be grown-up, socially for pleasure, and for escapism. The alcoholic, problem drinker and the drug addict are still in the minority, but the problem drinker of today can become the drug addict of tomorrow. The only real answer to this ever growing area of abuse is better education and better guidance for all at a much earlier age. Prevention must be better than cure.

\section{REFERENCES}

Denney, R. C. (1982). In Getting the Most Out of Food, pp. 65-9o. Burgess Hill: Van den Berghs \& Jurgens.

Hawker, A. (1978). Adolescents and Alcohol. New York: B. Edsall \& Co.

Jenkins, S. A. (1982). In Getting the Most Out of Food, pp. 35-63. Burgess Hill: Van den Berghs $\&$ Jurgens.

Lelbach, W. K. (1972). Deutsch Medizin Wissenschaft 97, 1435-1437.

Lieber, C. S. (1980). British fournal on Alcohol and Alcoholism 15(3), 95-103.

National Children's Bureau (1979). Highlight, no. 37, 1-2. London: National Children's Bureau.

National Council of Women of Great Britain (1980). Alcohol and the Unborn Child, the Fetal Alcohol Syndrome, pp. 1-20. London: National Council of Women of Great Britain.

Parliamentary All-Party Penal Affairs Group (1983). The Prevention of Crime Among Young People, pp. 78-87. London: H.M. Stationery Office.

Plant, M. A., Peck, D. F. \& Stuart, R. (1982). British fournal of Addiction 77, 75-90.

Ritson, B. (1 983). Child Health, vol. 2, pp. 108-1 18 , Basel: Karger.

Rosett, H. L., Weiner, L., Zuckerman, B., McKinlay, S. \& Edelin, K. C. (1980). Alcoholism: Clinical and Experimental Research, 4, 178-184.

Royal College of Psychiatrists (1979). Alcohol and Alcoholism, London: Tavistock Publications.

Smith, R. (1981). British Medical fournal, 31 st October, 43-45.

Staisey, N. L. \& Fried, P. A. (1983). Fournal of Studies on Alcohol 44(2), 262-270.

Tindle, E. (1983). National Council on Alcoholism News Autumn, 4. London: National Council on Alcoholism.

Wernicke, C. (188I). Lehrbuch Gehirnkrankh 2, 229-242.

Willet, W., Hennekens, C. H., Siezel, A. J., Adner, M. M. \& Castelli, W. P. (1980). New England Yournal of Medicine 20, 3103.

World Health Organization (1957). Evaluation of Dependence Producing Drugs. Report of the Expert Committee On Drugs Liable To Cause Addiction. Technical Report Series No. 287. Geneva: WHO. 